\title{
OBSERVATIONS OF THE PLASMA FLOW IN COMET P/SWIFT-TUTTLE
}

\author{
Michael E. Brown, Christopher M. Johns, and Hyron Spinrad
}

Department of Astronomy, University of California at Berkeley

\begin{abstract}
We present direct ground based observations of the plasma flow sunward and tailward of the nucleus of comet $\mathrm{P} / \mathrm{Swift}$-Tuttle. The observations are long-slit high resolution spectra of the $\mathrm{H}_{2} \mathrm{O}^{+}$emission centered at $6199 \AA$ with a velocity resolution of about $7 \mathrm{~km} \mathrm{~s}^{-1}$ (FWHM) and a spatial resolution of about $10^{4} \mathrm{~km}$ at the comet. Emission is visible from just inside the predicted position of the cometopause on the sunward side of the nucleus out to $5 \times 10^{5} \mathrm{~km}$ on the tailward side. The deceleration of the solar plasma on the sunward side is clearly observed as is the acceleration of cometary ions into the tail. These observations show the effectiveness of ground based methods for the systematic study of cometary plasmas and point to the need for a better theoretical understanding of their acceleration mechanisms.
\end{abstract}

\section{Introduction}

Our understanding of cometary plasmas was greatly increased by the knowledge gained from the in situ measurements obtained by the spacecraft armada to comets Halley and Giacobini-Zinner. But while spacecraft provide a wealth of information, the information is usually limited to a snap shot of a single object at a single instant. Use of ground-based observation allows the long term study of individual comets and the systematic study of the entire class, but ground based measurements of plasma properties in comets have traditionally been difficult and have not been detailed enough to allow the study of important physical regimes. For three decades, the plasma velocities in the tails of comets have been studied from the ground through direct observations of the proper motions of features in the tails. For example, Jockers [1981] compared sequential images of comet Kohoutek and measured the motions of coherent features which he could discern in multiple images. This method is difficult because the velocity can only be measured at locations along the tail where these features are visible, and the mechanism producing the features may also affect the plasma flow. Direct doppler measurement of the tail velocities was first achieved by Huppler et al. [1975] for comet Kohoutek and was systematically used for the study of plasma velocities in comet Halley by Scherb et al. [1990]. These doppler measurements used a highresolution scanning Fabry-Perot spectrometer to isolate the $6199 \AA \mathrm{H}_{2} \mathrm{O}^{+}$emission and to detect the small velocity shifts in the emission. To date, the use of this Fabry-Perot method has required a large entrance aperture to gather sufficient light, so use of this method has not allowed the study of small-scale structure. The method of short-slit

\section{Copyright 1993 by the American Geophysical Union.}

Paper number 93GL01123

0094-8534/93/93GL-01123\$03.00 spectroscopy was also used for comet Halley [S. Wyckoff and E. Lindholm, private communication, 1993], but here the aperture is too small to allow the measurement of the plasma parameters in more than one small region at a time, so again anything other than the gross structure remains unresolved.

We present here results from the first ground-based observations with sufficient velocity and spatial resolution to allow continuous measurement of velocity structures over a wide range of spatial scales. In the next section we describe the observational method and the details of the data reduction. The following section discusses the implications of the plasma velocity measurements, and we conclude with a few thoughts on strategies for future observations and a comment on the need for a different type of theoretical understanding.

\section{Observations}

The observations of plasma velocities in comet $\mathrm{P} /$ Swift-Tuttle were made using the 0.6 meter coudé auxiliary telescope coupled to the Hamilton echelle spectrograph at Lick Observatory between the nights of 23 November and 24 December 1992. To obtain both spectral and spatial information we used the method of long-slit spectroscopy which gave a spectrum at each position along the slit. The spectra were recorded by a Ford 2048 X 2048 CCD chip that was binned by a factor of four in the spatial dimension and a factor of two in the wavelength dimension to decrease the readout noise of the chip. The nucleus of the comet was placed near one edge of the slit, and the slit was then aligned along the tailward projection of the sun-comet vector. The observations achieved a spectral resolution of $\lambda / \delta \lambda($ FWHM $) \sim 40000$ (corresponding to a velocity resolution of about $7 \mathrm{~km} \mathrm{~s}^{-1}$ ) and a spatial resolution of about 10 arc seconds per binned pixel (at typical comet geocentric distances of about 1.3 $\mathrm{AU}, 10$ arc seconds projected to about $10^{4} \mathrm{~km}$ ) along a 6 arc minute slit (about $4 \times 10^{5} \mathrm{~km}$ ). Exposure times were between 1800 and 3600 minutes, and the comet had an approximate observed visual magnitude of 5.0 during the period of observations. The heliocentric distance of the comet ranged from 1.01 to $0.98 \mathrm{AU}$.

The raw two-dimensional spectra were reduced identically: first, the bias and dark currents were subtracted, and then the spectra were flat-fielded using the spectrum of a quartz lamp to map the pixel-to-pixel variations in the CCD. Non-uniformities in transmission along the spatial dimension of the slit were removed by dividing the spectra by the spatial distribution measured in a day sky spectrum. The wavelength scale for the spectra was calibrated from spectra of a thorium-argon lamp with a high density of known spectral lines. The wavelength scale of each spectrum was then shifted to account for the earth-comet velocity, which was between 21 and $28 \mathrm{~km} \mathrm{~s}^{-1}$, by placing the $\mathrm{H}_{2} \mathrm{O}^{+}$emission lines of the nucleus at the known $\mathrm{H}_{2} \mathrm{O}^{+}$rest emission wavelength. Figure 1 shows the two-dimensional spectrum from the 


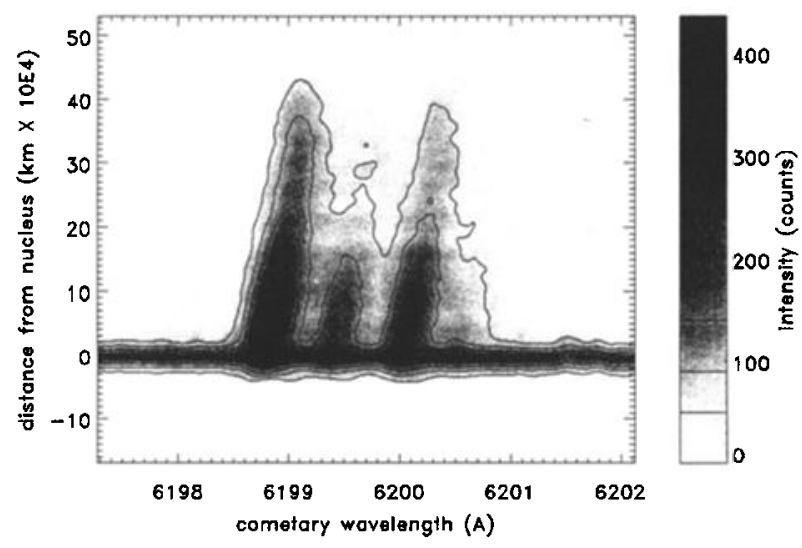

Fig. 1. A long slit $\mathrm{H}_{2} \mathrm{O}^{+}$spectrum of Comet Swift-Tuttle from the night of 26 November 1992. The acceleration of the plasma tail is clearly visible in this spectrum as the shift to longer wavelengths of the $\mathrm{H}_{2} \mathrm{O}^{+}$emission. The spectrograph slit was aligned along the sun-comet vector, so the spatial dimension of the spectrum gives distance from the nucleus of the comet along this suncomet vector. The continuous emission at the position of the nucleus is solar continuum light scattered from cometary dust particles. At this wavelength, a doppler shift of $1 \AA$ corresponds to a velocity of $48 \mathrm{~km} \mathrm{~s}^{-1}$.

night of 30 November 1992. The emission from $\mathrm{H}_{2} \mathrm{O}^{+}$is clearly visible as the four slanted emission lines; the slant of the lines is caused by the doppler shifts that it is our goal to measure. The continuous emission at the nucleus is caused by sun light scattered from the dust coma of the comet. Figure 2 compares the spectra at three points along the tail of the comet and shows the excellent signalto-noise achieved in these observations.

To obtain precise velocity measurements from the spectra we used a cross-correlation method where the spectrum at each spatial location is cross-correlated with the spectrum at each other location to obtain the velocity shift. From this system of mutual cross-correlations we obtained a best-fit for the velocity shift at each spatial location. For each spectrum, we determined the errors in this method by using the same cross-correlation method on a hundred simulated two-dimensional spectra having the same velocity shift and random noise at a level determined by the measured signal-to-noise at each location in the real spectrum. Because of the high signal-to-noise of these observations, this cross-correlation method allowed determination of relative velocities to a one sigma accuracy of better than $1 \mathrm{~km} \mathrm{~s}^{-1}$.

Although these observations represent a column integration through the coma rather than a single measurement along the sun-comet line, we believe that because of the quasi-cylindrical geometry and central brightness concentration of the emission, the column integrated velocity is typical of the velocity in the plane of the sky. To correct the velocity to that along the sun-comet vector we divided the measured velocity by the cosine of the sun-comet-earth angle to determine the component along this vector. Similarly, we divided the distance along the plane of the sky by the sine of this angle to determine the distance along this vector. Figure 3 shows the results of the measurements of the velocity shifts for the six best spectra and lists the time of center exposure for each spectrum.
Flow on the Sunward Side

As neutral water molecules sublimate from the cometary nucleus and flow outward, they eventually ionize into $\mathrm{H}_{2} \mathrm{O}^{+}$or dissociate into smaller fragments which may themselves become ionized. The newly created ions are picked up by the solar wind flow and quickly accelerated to the local solar wind velocity. Thus measurements of the cometary plasma flow are equivalent to measurements of the solar wind plasma flow at that location in the comet. When encountering a comet, the solar wind flow goes through three boundaries on the sunward side of the cometary nucleus: the bow shock, the cometopause, and the ionopause [Flammer 1991]. Outside of the cometary bow shock the solar wind flows mostly undisturbed, at a typical velocity of about $400 \mathrm{~km} \mathrm{~s}^{-1}$. Inside of the bow shock, the solar wind begins interacting with cometary ions. In this region, mass-loading of these ions causes the flow speed of the solar wind to decrease. At the cometopause, the neutral density has increased to the point where collisions with outflowing neutral cometary particles decelerates the solar wind even more quickly. As the density of neutrals continues to increase, the solar wind (now heavily contaminated with cometary pick-up ions) eventually reaches the ionopause where strong collisional coupling between cometary neutrals and ions completely excludes the solar wind plasma.

Flammer [1991] has estimated the locations of these boundaries for Halley-type comets. Preliminary observations indicate that $S$ wift-Tuttle had a production rate almost comparable to that of Halley at the 1986 encounters [A'Hearn 1992], thus we will consider the Halley estimates approximately appropriate for comet P/SwiftTuttle. The estimates predict the bow shock around $5 \times 10^{5} \mathrm{~km}$, the cometopause around $5 \times 10^{4} \mathrm{~km}$, and the ionopause around $4000 \mathrm{~km}$.

Figure 3 shows the measured plasma velocities on the sunward side of the nucleus. All detections of cometary jons are inside of the predicted location of the bow shock and also inside of the predicted location of the cometopause. With the modest spatial resolution achieved, however, the region of the ionopause remains unresolved. As expected from the basic scenario outlined

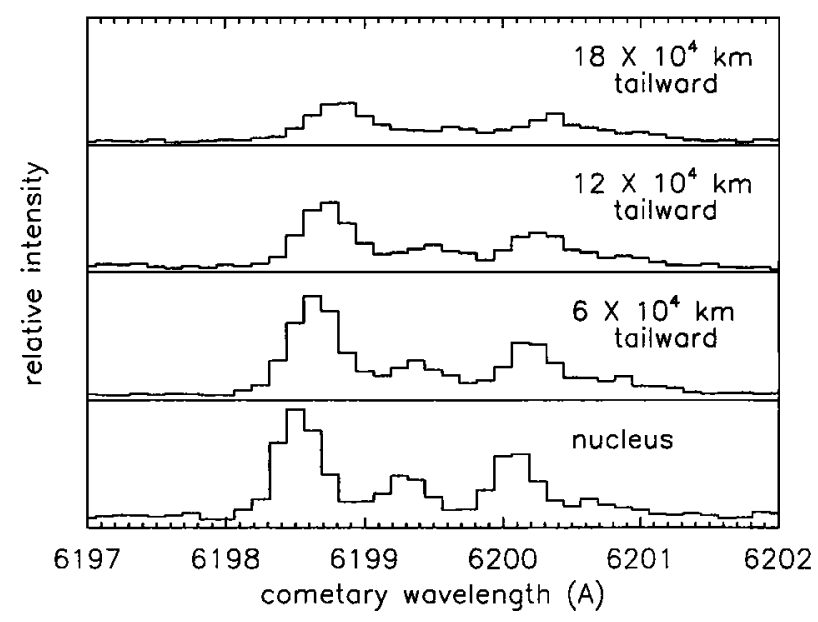

Fig. 2. One dimensional spectra at four distances from the nucleus. The velocity shift of the spectra is clearly visible in these plots, as is the high signal-to-noise of these observations. The plasma temperature can be measured from the line width of these emissions. 


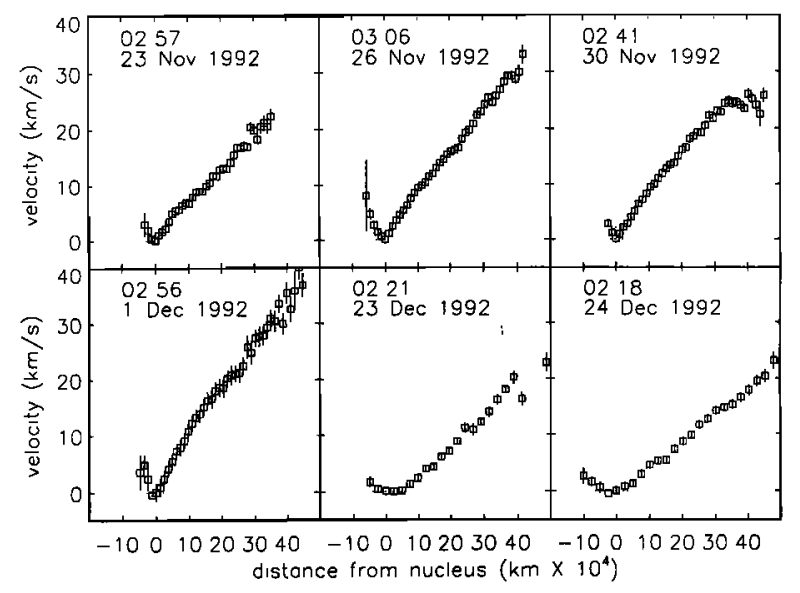

Fig. 3. Velocity shifts measured from the six best cometary spectra. These shifts were calculated using a cross-correlation method and corrected for projection effects. The error bars shown are one sigma errors determined through a Monte Carlo method detailed in the text. Negative distances are sunward of the nucleus, while positive distances are tailward, and the vertical dotted line denotes the position of the nucleus. The date and time (UT) of the mid-point of the observation are shown. Note the time variability of the tail velocities and the good measurement of the sunward velocities.

above, the measured flow of $\mathrm{H}_{2} \mathrm{O}^{+}$ions and thus also of solar wind ions inside of the predicted position of the cometopause is already significantly slowed from the $\sim 400 \mathrm{~km} \mathrm{~s}^{-1}$ expected outside of the bow shock. Typical measured velocities at $5 \times 10^{4} \mathrm{~km}$ sunward are around $5 \mathrm{~km} \mathrm{~s}^{-1}$. Giotto measured velocities of about $10 \mathrm{~km} \mathrm{~s}^{-1}$ at this distance, and did not measure velocities as low as $5 \mathrm{~km} \mathrm{~s}^{-1}$ until a distance of $2.5 \times 10^{4} \mathrm{~km}$ [Goldstein 1992], though this difference of a factor of two could be simply accounted for by the fact that Giotto did not travel along the sun-comet line. MHD modeling of the Halley plasma flow predicts velocities of around $15 \mathrm{~km} \mathrm{~s}^{-1}$ at a distance of $5 \times 10^{4} \mathrm{~km}$ sunward [Schmidt and Wegmann 1991]. The discrepancies between the observed and modeled flows could perhaps point to an increase in the efficiency of the neutral coupling to the solar wind at distances further than the predicted position of the cometopause.

The difficulty of measuring this flow from the ground is apparent: the velocity changes quickly within a small distance as the emission intensity is dropping quickly. Even with the current long-slit technique we have not spatially resolved the inner regions of the coma, nor have we detected the predicted drastic deceleration at the cometopause. In the last section of this Letter we will discuss prospects for obtaining spectra with higher spatial resolution and extending the plasma measurement further sunward.

\section{Flow on the Tailward Side}

The details of the near-nuclear plasma flow in the tailward directions of comets are not as well understood as those of the flow on the sunward side. Although much information was gained about the more distant tail regions by the ICE spacecraft encounter with comet Giacobini-Zinner, the tail side of the ionopause and nearnuclear regions was not encountered by any spacecraft.
MHD simulations have yet to reach a self-consistent solution for this region [Neubauer 1991], and no previous ground-based observation has had sufficient velocity and spatial resolution to discern this region. Our high resolution long-slit spectra provide the first contiguous and detailed look at this region of a comet.

Examination of the near-nuclear velocities on the tailward side of the nucleus in figure 3 shows a smooth transition from the low velocity flow near the cometary nucleus into the tail region. In each spectrum the tailward acceleration starts at the first measured point past the nucleus, thus the tailward extent of the low velocity ionosphere is restricted to a distance of less than about $10^{4} \mathrm{~km}$. The MHD models of Schmidt and Wegman [1991] predict a teardrop shape to the ionopause, with the tailward section extending to around $2 \times 10^{4} \mathrm{~km}$. Because of the stretching on the tailward side we do have sufficient spatial resolution to view into this region of the ionopause, and though our observations are a column integration through the comet and the view is perhaps distorted by plasma flow on the outside of the ionopause, our observations indicate a smaller tailward ionopause than currently predicted. In addition, at a distance of about $5 \times 10^{4} \mathrm{~km}$, where we measure velocities of around $5 \mathrm{~km} \mathrm{~s}^{-1}$, the numerical models [e.g. Schmidt and Wegman 1991] predict much higher velocities of around $15 \mathrm{~km} \mathrm{~s}^{-1}$. This region is one that is difficult to study numerically, observationally, or in situ, so it is important to develop this technique of probing the velocities in this region of comets.

Further down the tail, at a distance approximately comparable for this comet to the location of the crosstail trajectory of the ICE spacecraft for comet GiacobiniZinner, a possible discontinuity in the slope of the distance-velocity curve is apparent. This discontinuity is best observed in the spectrum from 1 December 1992 at a distance of about $15 \times 10^{4} \mathrm{~km}$, and it can also be seen between 10 and $40 \times 10^{4} \mathrm{~km}$ in most of the other spectra. The discontinuity is better observed in the acceleration, plotted in figure 4. These accelerations were determined by smoothing the velocities in figure 3 and calculating the instantaneous acceleration at each point. The velocity slope discontinuity is the point where the slope of the acceleration suddenly decreases. For the spectrum of 30 November 1992 the acceleration itself even begins to decrease and goes to zero. These discontinuities could be due to more slowly accelerating discrete structures in the tail and not actually represent changes in the bulk flow. In this case we would expect to see these structures in images of the plasma tail. In the future we hope that simultaneous spectroscopy and imaging of the plasma tail will reveal the nature of these features.

Near the end of the observable tail still another change in the acceleration is visible. In three of the spectra, at a distance of about $3 \times 10^{5} \mathrm{~km}$ the acceleration begins once more to increase quickly. The MHD simulations are not enlightening at this point: Schmidt and Wegman [1991] predict a smoothly varying velocity of around $60 \mathrm{~km} \mathrm{~s}^{-1}$ at this point, while the measured velocities are only around 20 to $30 \mathrm{~km} \mathrm{~s}^{-1}$. Measurements of the flow in comet Halley at this distance by Scherb et al. [1990] show that the velocity is highly time variable, ranging between about 20 and $60 \mathrm{~km} \mathrm{~s}^{-1}$, but again the Halley measurements do not have sufficient spatial resolution to discern the discontinuity in the acceleration. The physical mechanisms responsible for the acceleration discontinuities are unknown, but we hope 


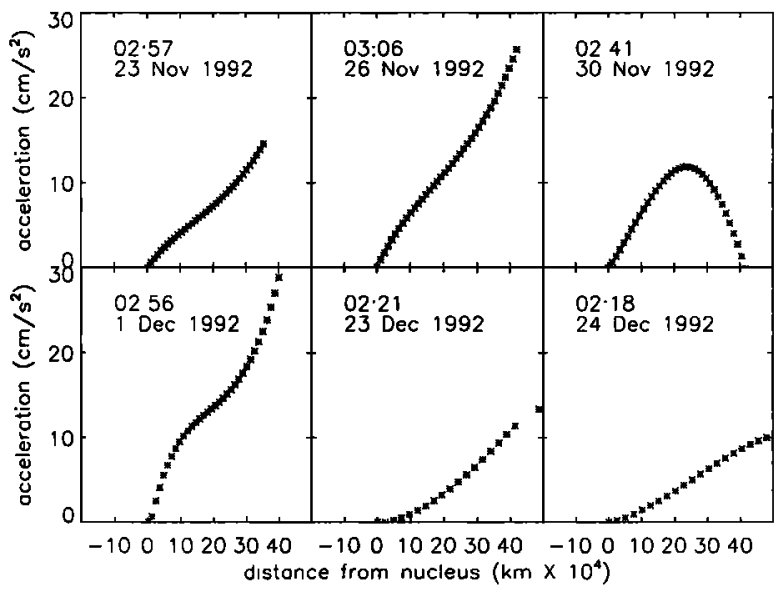

Fig. 4. Accelerations measured from the velocity shifts in the six best cometary spectra. The accelerations were calculated by smoothing the measured velocities and then determining the instantaneous acceleration at each point. Note the discontinuities in most of the measured accelerations between 10 and $20 \times 10^{4} \mathrm{~km}$ and between 30 and $40 \times 10^{4} \mathrm{~km}$.

that these observations inspire theoretical inquiries into these processes.

\section{Conclusions}

We have shown that it is possible to obtain high quality cometary plasma data from the ground. Although the current observations do not have sufficient spatial resolution to resolve the ionopause region, nor do they extend far enough in the sunward direction to study the predicted cometopause transition, the observational techniques can be extended to allow both of these possibilities. The spatial resolution achievable with our instrument was approximately 9 arc seconds, but for a brighter comet we could achieve a factor of four higher spatial resolution by not binning the CCD chip. For comet Swift-Tuttle, this increase would give a resolution of about $2500 \mathrm{~km}$, allowing direct observation of the ionosphere. Obtaining data outside of the cometopause is more difficult, but it is possible that an observational emphasis on the sunward rather than tailward side of the nucleus would allow detection of these $\mathrm{H}_{2} \mathrm{O}^{+}$emissions.

Ground based cometary observations like these will remain the best method of systematic study of comets, for although they generate neither the excitement nor the wealth of data of a spacecraft encounter, they are the only current method that allows both the long term study of single comets and systematic study of comets as a class. And with the prospects for any new in situ measurements in the near future quite small, ground based observation will remain not just the best, but the only method of studying comets at all.

Interpretation of measurements such as these from complicated objects like comets is difficult at best. All too often (as is frequently the case for this paper) the interpretation is simply a comparison between the observations and numerical models, showing where the model does and does not agree with the observations, and since the models are quite complex and comet-specific; even this method is not usually possible except for a small number of comets. If we are to study comets as a class, we must have truly generalizable theories to which we can compare the data and make appropriate interpretations. We therefore urge the continued development of both systematic ground based observation and the theory with which to interpret these new data.

Acknowledgements. We are indebted to Tony Misch at Lick Observatory for his continued assistance and to Don Yeomans for an updated ephemeris. MEB acknowledges the support of a NASA Graduate Fellowship, and HS is supported by NASA grant NAGW-2883.

\section{References}

A'Hearn,M., Periodic Comet Swift-Tuttle (1992t), IAU Circular, 5663, 1992.

Flammer, K.R., The global interactions of comets with the solar wind, in Comets in the Post-Halley Era, eds. R.L. Newburg et al., 1125-1144, Kluwer Academic Publishers, Dordrecht, 1991.

Goldstein, B.E., R. Goldstein, M. Neugebauer, S.A. Fuselier, E.G. Shelley, H. Balsiger, G. Kettmann, W.-H. Ip, H. Rosenbauer, and R. Schwenn, Observations of plasma dynamics in the coma of $\mathrm{P} /$ Halley by the Giotto ion mass spectrometer, $J$. Geophys. Res., 97, 4121, 1992.

Huppler, D., R.J. Reynolds, F. Roesler, F. Scherb, and J. Trauger, Observations of the Comet Kohoutek (1973f) with a ground-based Fabry-Perot spectrometer, Astrophys. J., 202, 276, 1975.

Jockers, K., Plasma dynamics in the tail of Comet Kohoutek 1973 XII, Icarus,47, 397, 1981.

Neubauer, F.M., The magnetic field structure of the cometary plasma environment, in Comets in the Post-Halley Era, eds. R.L. Newburg et al., 1107-1124, Kluwer Academic Publishers, Dordrecht, 1991.

Schmidt, H.U. and R. Wegman, An MHD model of cometary plasma and comparison with observations, in Cometary Plasma Processes, ed. A.D. Johnstone, 49-63, AGU Publications, Washington, D.C., 1991.

Scherb, F., K. Magee-Sauer, F.L. Roesler, and J. Harlander, Fabry-Perot observations of Comet Halley $\mathrm{H}_{2} \mathrm{O}^{+}$, Icarus, 86, 172, 1990.

M. Brown, C. Johns, and H. Spinrad, Department of Astronomy, University of California, Berkeley, CA 94720.

(Received: March 22, 1993;

Accepted: April 16, 1993) 